\title{
The Relation among the Solar Activity, the Total Ozone, QBO, NAO, and ENSO by Wavelet-Based Multifractal Analysis
}

\author{
Fumio Maruyama \\ Faculty of Human Health Science, Matsumoto University, Matsumoto, Japan \\ Email: fmaruya@nagoya-u.jp
}

How to cite this paper: Maruyama, $F$. (2018) The Relation among the Solar Activity, the Total Ozone, QBO, NAO, and ENSO by Wavelet-Based Multifractal Analysis. Journal of Applied Mathematics and Physics, 6, 1301-1314.

https://doi.org/10.4236/jamp.2018.66109

Received: May 21, 2018

Accepted: June 25, 2018

Published: June 28, 2018

Copyright $\odot 2018$ by author and Scientific Research Publishing Inc. This work is licensed under the Creative Commons Attribution International License (CC BY 4.0).

http://creativecommons.org/licenses/by/4.0/

c) (i) Open Access

\begin{abstract}
There is an increasing interest in the relation between the solar activity and climate change. As for the solar activity, a fractal property of the sunspot number was studied by many works. In general, a fractal property was observed in the time series of dynamics of complex systems. The purposes of this study are to investigate the relations among the solar activity, total ozone, Quasi-Biennial Oscillation (QBO), the North Atlantic Oscillation (NAO), and El Niño-Southern Oscillation (ENSO) from a view of multi-fractality. To detect the changes of multifractality, we examined the multifractal analysis on the time series of the solar 10.7-cm radio flux (F10.7 flux), total ozone, QBO, NAO, and Niño3.4 indices. During the period 1950 and 2010, for the F10.7 flux and QBO index, the matching in monofractality or multifractality is observed and the increase and decrease of multifractality is similar; that is the change of multifractality is similar. In the same way, it is very similar, during the period 1985 and 2010, for the QBO and the total ozone, and during the period 1950 and 2010, for the QBO, and NAO and for the QBO, and Niño3.4. Compared to Niño3.4, the multifractality of $\mathrm{NAO}$ and $\mathrm{QBO}$ was strong and it turns out that they are undergoing unstable change. The relation among the solar activity, total ozone, QBO, NAO, and ENSO was clarified by the methods of fractal analysis and the wavelet coherence. These findings will contribute to the research of the relation between the solar activity and climate change.
\end{abstract}

\section{Keywords}

Solar Radio Flux, Total Ozone, QBO, NAO, ENSO, Wavelet, Multifractal

\section{Introduction}

Various objects in nature show the so-called self-similarity or fractal property. 
Monofractal shows an approximately similar pattern at different scales and is characterized by a fractal dimension. Multifractal is a non-uniform, more complex fractal and is decomposed into many sub-sets characterized by different fractal dimensions. Fractal property can be observed in the time series representing dynamics of complex systems as well. A change of fractality accompanies a phase transition and changes of state. The multifractal properties of daily rainfall were investigated in two contrasting climates: an East Asian monsoon climate with extreme rainfall variability and a temperate climate with moderate rainfall variability [1]. In two contrasting climates, the frontal rainfall shows monofractality and the convective-type rainfall shows multifractality.

Hence, climate change can be interpreted from the perspective of fractals. A change of fractality may be observed when the climate changes. We attempt to explain changes in climate, referred to as regime shifts, by analyzing fractality. We use the wavelet transform to analyze the multifractal behavior of the climate index. Wavelet methods are useful for the analysis of complex non-stationary time series. The wavelet transform allows reliable multifractal analysis to be performed [2]. In our previous paper [3], in terms of the multifractal analysis, we conclude that a climatic regime shift corresponds to a change from multifractality to monofractality of the Pacific Decadal Oscillation (PDO) index.

The influence of solar activity on climate has been discussed for a long time.

Recent measurements from space indicate that the total solar irradiance changes associated with the 11-year solar cycle are negligibly small (0.1\%), although larger $(4 \%-8 \%)$ variations are found in the ultraviolet (UV) range of $200-250 \mathrm{~nm}$ [4]. Even if we do not expect direct solar cycle impacts at Earth's surface, a significant influence should be detected in the stratopause region [5]. The influence of the solar activity on the climate has been studied as below.

A decadal variation that correlates positively with 11-year solar activity cycle of tropical lower stratospheric ozone and temperature has previously been identified.

However, the El Niño-Southern Oscillation (ENSO) also influences lower stratospheric ozone and temperature [6]. The coherent temperature and ozone signals are evidence that ENSO modulates upwelling in the tropical lower stratosphere [7].

Solar and the Quasi-Biennial Oscillation (QBO) in the equatorial lower stratosphere influences on the timing of stratospheric sudden warmings [8]. The influence of the 11-year solar cycle on the QBO was shown [9], [10]. Chemical constituents, such as ozone, water vapor, and methane, are affected by circulation changes induced by the QBO [11].

A significant part of temperature variation could be the result of a solar wind interaction with the Earth's atmosphere and a subsequent modulation of the North Atratic Ocillation (NAO) [12].

The relations between the solar activity and climate indices and those between the climate indices have been examined, but the relations of their fractal properties have not been investigated. So in this study, we examined the relation among 
the solar activity, the total ozone, QBO, NAO, and ENSO by the change of fractality paying attention to the $\mathrm{QBO}$. To detect the changes of multifractality, we examine the multifractal analysis on the solar $10.7-\mathrm{cm}$ radio flux (F10.7 flux), QBO, NAO, Niño3.4 indices, the Southern Oscillation Index (SOI), and total ozone using the wavelet transform. Moreover we examined the wavelet coherence and phase of those indices. To investigate the influence of the sun on the climate in the case of the strong solar activity, we examined the wavelet coherence and phase between them, and the change of fractality.

\section{Data and Method of Analysis}

We used the monthly time series provided by NOAA's Climate Prediction Center, USA (CPC), as detailed below. We used the solar 10.7-cm radio flux (F10.7 flux) (Figure 1(a)), which is an excellent indicator of the solar activity, QBO (Figure 1(b)), NAO (Figure 1(c)), Niño3.4 (Figure 1(d)) indices and SOI. QBO index from the zonal average of the $30 \mathrm{mb}$ zonal wind at the equator was used. The total ozone provided by NASA (nasasearch.nasa.gov) were used.

We used the Daubechies wavelet as the analyzing wavelet because it is widely used in solving a broad range of problems, e.g., self-similarity properties of a signal or fractal problems and signal discontinuities. The data used were a discrete signal that fitted the Daubechies Mother wavelet with the capability of precise inverse transformation. Hence, precisely optimal value of $\tau(q)$ could be calculated as explained below. We then estimated the scaling of the partition function $Z_{q}(a)$, which is defined as the sum of the $q$-th powers of the modulus of the wavelet transform coefficients at scale a. In our study, the wavelet-transform coefficients did not become zero, and therefore, for a precise calculation, the summation was considered for the entire set. Muzy et al. [2] defined $Z_{q}(a)$ as the sum of the $q$-th powers of the local maxima of the modulus to avoid division by zero. We obtained the partition function $Z_{q}(a)$ :

$$
Z_{q}(a)=\sum\left|W_{\varphi}[f](a, b)\right|^{q}
$$

where $W_{\varphi}[f](a, b)$ is the wavelet coefficient of the function $f$, $a$ is a scale parameter and $b$ is a space parameter. The time window was set to six years for the following outlined reasons. We calculated the wavelets using a time window of various periods, 10, 6 and 4 years. For a time window of 10 years, a slow change of fractality was observed. Thus, this case was inappropriate to find a rapid change of climate indices because when we integrated the wavelet coefficient over a wide range, small changes were canceled. For four years, a fast change of fractality was observed. The overlap of the first and subsequent data was 3 years, which is shorter than the 9 years in the case of the 10-year calculation and thus the change of fractality was large. For six years, a moderate change of fractality was observed and hence the time window was set to this period. For small scales, we expect

$$
Z_{q}(a) \sim a^{\tau(q)}
$$




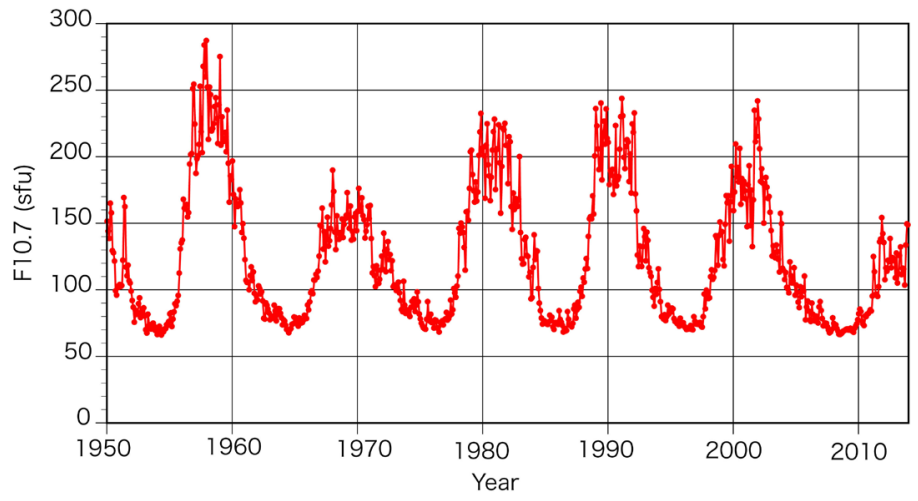

(a)

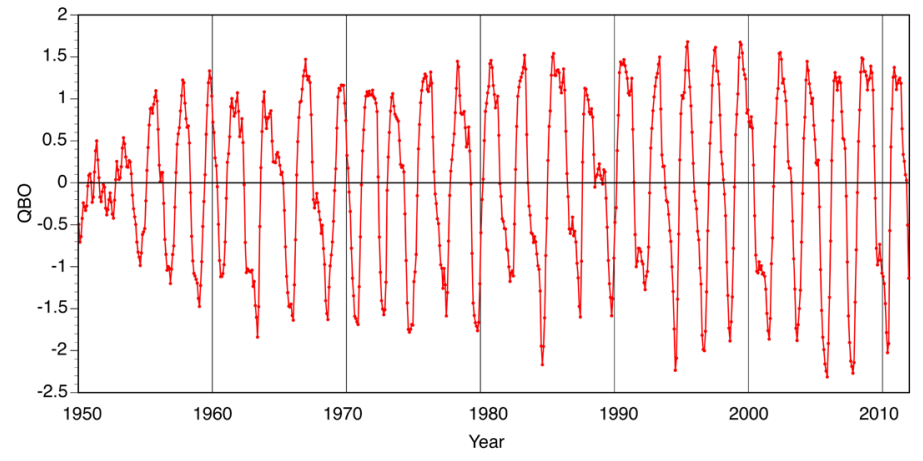

(b)

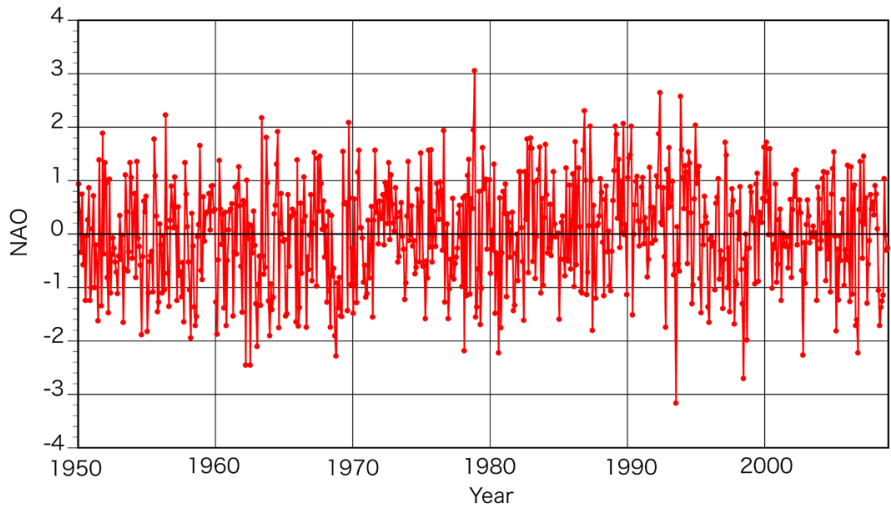

(c)



(d)

Figure 1. (a) F10.7 flux; (b) QBO; (c) NAO; (d) Niño3.4. 
First, we investigate the changes of $Z_{q}(a)$ in time series at a different scale $a$ for each $q$. A plot of the logarithm of $Z_{q}(a)$ against the logarithm of time scale a was created. Here $t(q)$ is the slope of the linear fitted line on the log-log plot for each $q$. Next, we plot $\tau(q)$ vs $q$. The time window was then shifted forward one year and the process repeated. We define monofractal and multifractal as follows: if $\tau(q)$ is linear with respect to $q$, then the time series is said to be monofractal: if $\tau(q)$ is convex upwards with respect to $q$, then the time series is classified as multifractal [13]. We define that the value of $\mathrm{R}^{2}$, which is the coefficient of determination, for fitting straight line, if $\mathrm{R}^{2} \geq 0.98$ the time series is monofractal and if $0.98>\mathrm{R}^{2}$ that is multifractal.

We calculated $\tau(q)$ of different moments $q$ for individual records for the Niño3.4 index. In Figure 2, $\tau(q)$ between 1980 and 1994 is shown. The data were analyzed in six-year sets, e.g., $\tau(q)$ of n80 was calculated for the 1980-1985 period, and that of n81 was calculated for the 1981-1986 period. To examine the change of fractality, the time window was then shifted forward one year and $\tau(q)$ was calculated from n80 up to n89. A monofractal signal would correspond to a straight line for $\tau(q)$, while $\tau(q)$ would be nonlinear for a multifractal signal. Most of the multifractality observed is due to the negative value of $q$, i.e., small fluctuations are more inhomogeneous than large fluctuations. In Figure 2, the data sets were monofractal in the cases of n80 - n82, n85 - n87, and n89 and were multifractal in the cases of $\mathrm{n} 83, \mathrm{n} 84$, and $\mathrm{n} 88$.

We plot the value of the $t(-6)$ in each index. The negative large values of the $\tau(-6)$ show large multifractality. For the $\tau(-6), q=-6$ is the appropriate number to show the change of $\tau$. The value of the $\tau(-6)$ do not always correspond to the fractality obtained from the value of $\mathrm{R}^{2}$.

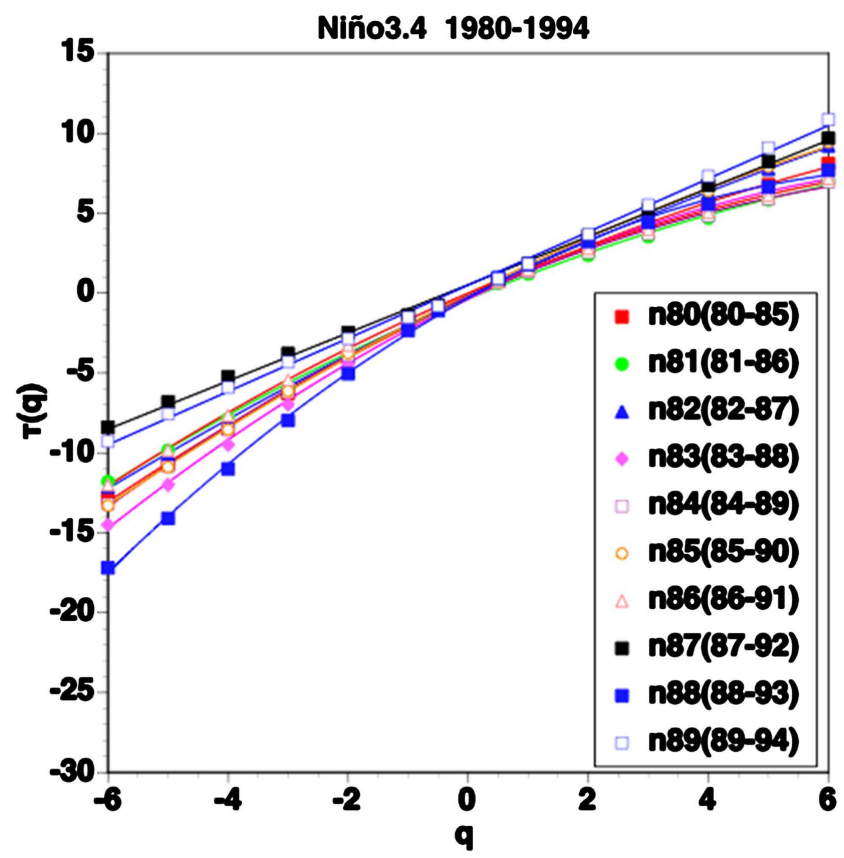

Figure 2. $\tau(q)$ for individual Niño3.4 between 1980 and 1994. 


\section{Results}

\subsection{The Influence of the Solar Radio Flux on the QBO}

To examine the influence of the solar activity on the QBO, we investigated the relation between the F10.7 flux and QBO index. The $t(-6)$ of the F10.7 flux and $\mathrm{QBO}$ index are shown in Figure 3. The red square shows monofractality and the green circle shows multifractality for the 6 years centered on the year plotted.

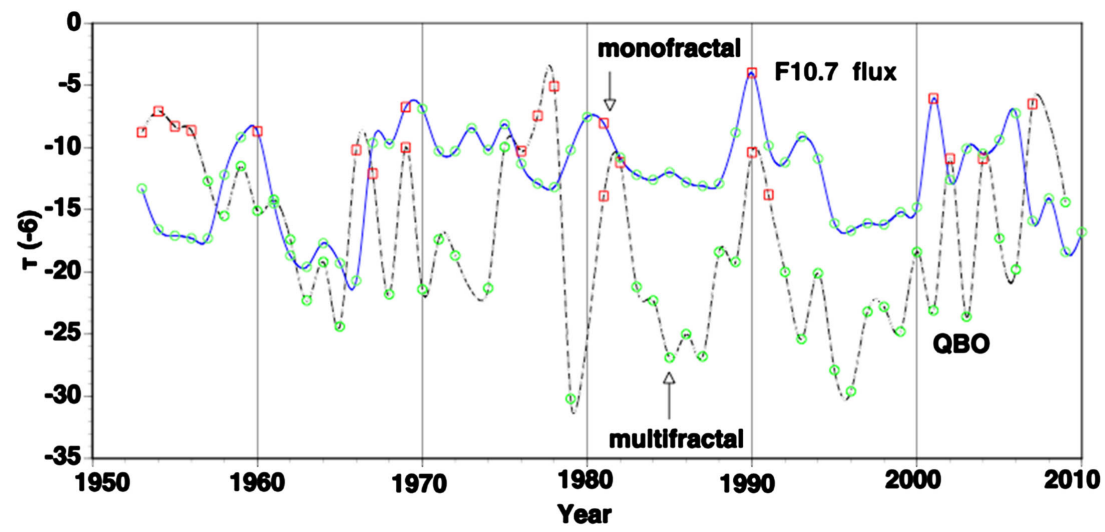

Wavelet Coherence
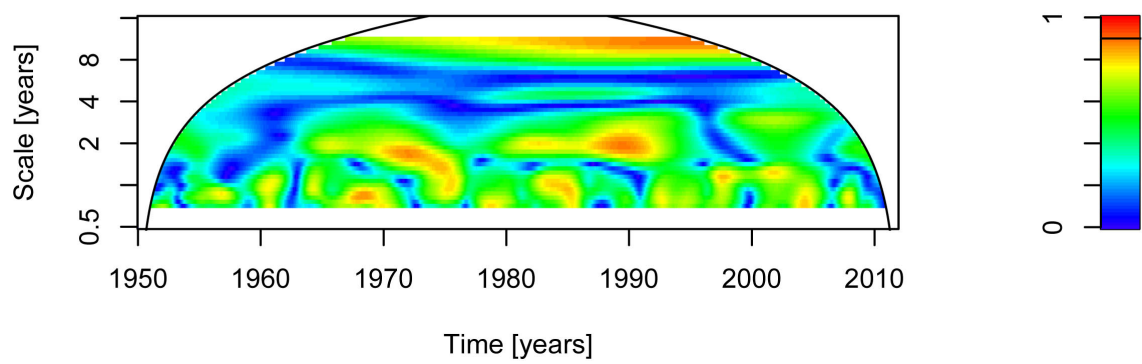

Phase
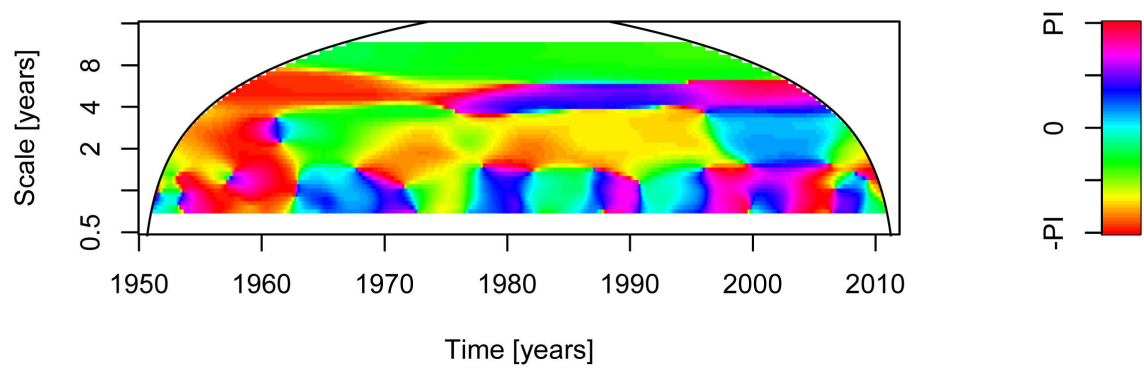

Figure 3. The $t(-6)$ of the F10.7 flux, and QBO index (top). Wavelet coherence (middle) and phase (bottom) between the F10.7 flux and QBO index. The thick black contour encloses regions of greater than $95 \%$ confidence. The thin black contour encloses regions of greater than $90 \%$ confidence. The cone of influence, which indicates the region affected by edge effects, is shown with a black line. In the wavelet phase, the positive value shown by the blue and pink shading means that the F10.7 flux leads QBO index and the negative value shown by the green, yellow and red shading means that QBO index leads the F10.7 flux. 
For example, the green circle for 1970 in the QBO shows multifractality between 1967 and 1972. The data was excluded from Figure 3 (top) for cases where we could not distinguish between monofractality and multifractality. During the period 1950 and 2010, for the F10.7 flux and QBO index, the matching in monofractality or multifractality is observed and the increase and decrease of multifractality are very similar, that is the change of multifractality is similar. We investigated the relation between the F10.7 flux and QBO index by means of wavelet coherence, phase and fractality. We show the wavelet coherence and phase using the Morlet wavelet between the F10.7 flux and QBO in Figure 3 (middle) and (bottom), respectively. The coherence between the F10.7 flux and QBO index in 1 - 2 year scale was strong and the lead and delay of the F10.7 flux was observed every ten years.

\subsection{Relation between the $\mathrm{QBO}$ and Total Ozone}

We investigated the relation between the QBO and total ozone. The $t(-6)$ of the QBO and total ozone are shown in Figure 4. During the period 1985 and 2010, for the QBO and total ozone, the change of multifractality are very similar. We show the wavelet coherence and phase using the Morlet wavelet between the QBO index and total ozone in Figure 4 (middle) and (bottom), respectively. The coherence between the QBO index and total ozone was strong in 2 - 3 year scale for 1980-1995 and in 1 year scale for 1995-2008 and the leads of the QBO index were observed. This result consisted with the result of [11].

\subsection{Relation between the NAO and QBO}

We investigated the relation between the NAO and QBO. The $\tau(-6)$ of the NAO and QBO are shown in Figure 5. During the period 1950 and 2010, for the NAO and $\mathrm{QBO}$, the change of multifractality is very similar. The coherence between the NAO and $\mathrm{QBO}$ indices was strong and the lead and delay of the QBO index was observed.

\subsection{Relation between the ENSO and QBO}

We investigated the relation between the ENSO and QBO. The $\tau(-6)$ of the Niño3.4 and QBO are shown in Figure 6. During the period 1950 and 2010, for the Niño3.4 and QBO, the change of multifractality is very similar. The coherence between the Niño3.4 and QBO indices was strong and the lead and delay of the QBO index was observed. The $\tau(-6)$ of the SOI and QBO are shown in Figure 7. During the period 1950 and 2010, for the SOI and QBO, the change of multifractality is very similar. The coherence between the SOI and QBO index was strong and the lead and delay of the QBO index was observed.

\section{Discussion}

We considered the relation among the solar activity, total ozone, QBO, NAO, and ENSO. We examined the changes of fractality, $t(-6)$ and the wavelet coherence and phase in those indices. 


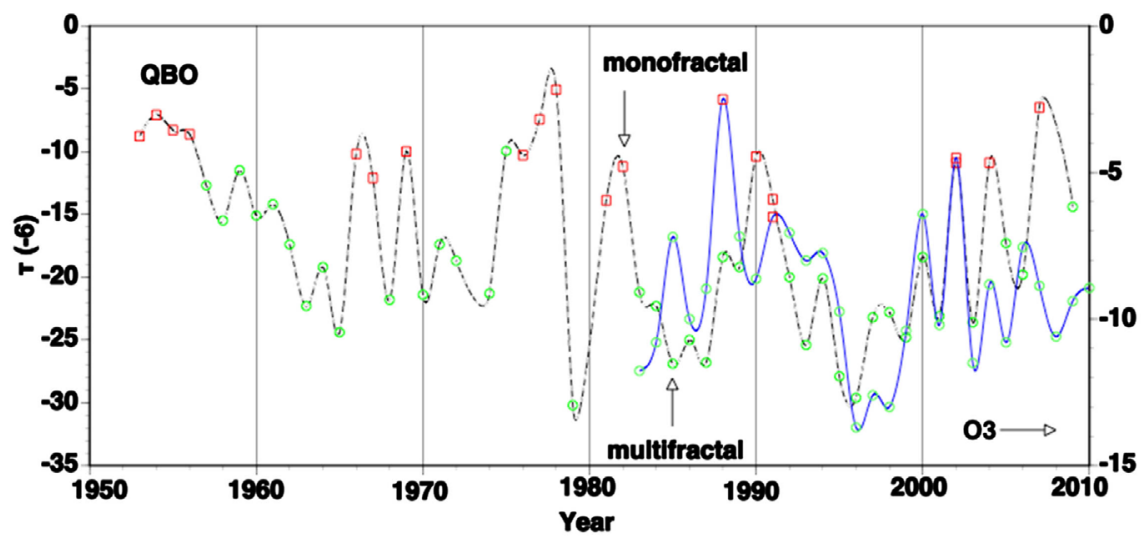

Wavelet Coherence

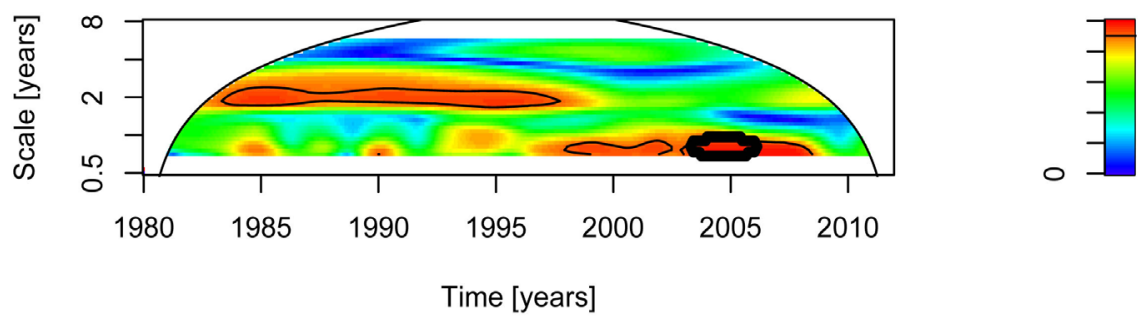

Phase

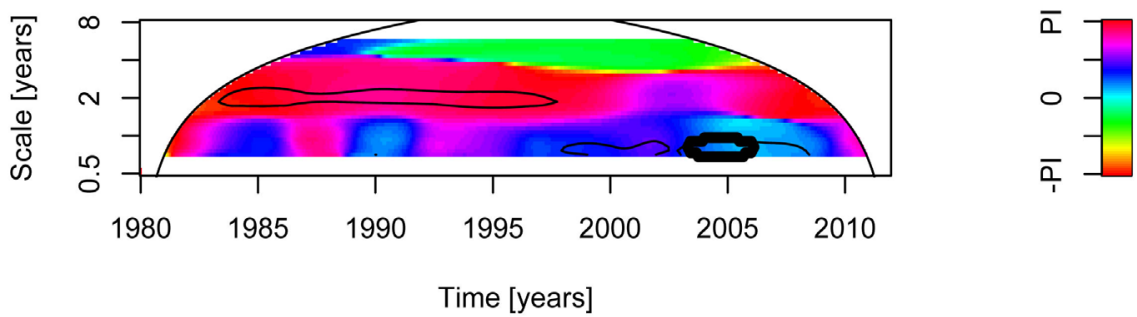

Figure 4. The $t(-6)$ of the QBO index and total ozone (top). Wavelet coherence (middle) and phase (bottom) between the QBO index and total ozone. In the wavelet phase, the positive value shown by the blue and pink shading means that the QBO leads the total ozone and the negative value shown by the green, yellow and red shading means that the total ozone leads the QBO.

During the period 1950 and 2010, for the F10.7 flux and QBO index, the matching in monofractality or multifractality is observed and the increase and decrease of multifractality are very similar, that is the change of multifractality is similar. The coherence between the F10.7 flux and QBO index in 1 - 2 year scale was strong and the lead and delay of the F10.7 flux was observed every ten years. The influence of the solar activity on the QBO has been shown. The influence of the 11-year solar cycle on the quasi-biennial Oscillation is shown [10]. As the correlation between $\mathrm{QBO}$ and F10.7 flux is strong, the QBO contains the 11-year cycle from the sun. The QBO influences the solar cycle in climate indices. Hence, the QBO influences on the F10.7 flux and leads the F10.7 flux. 


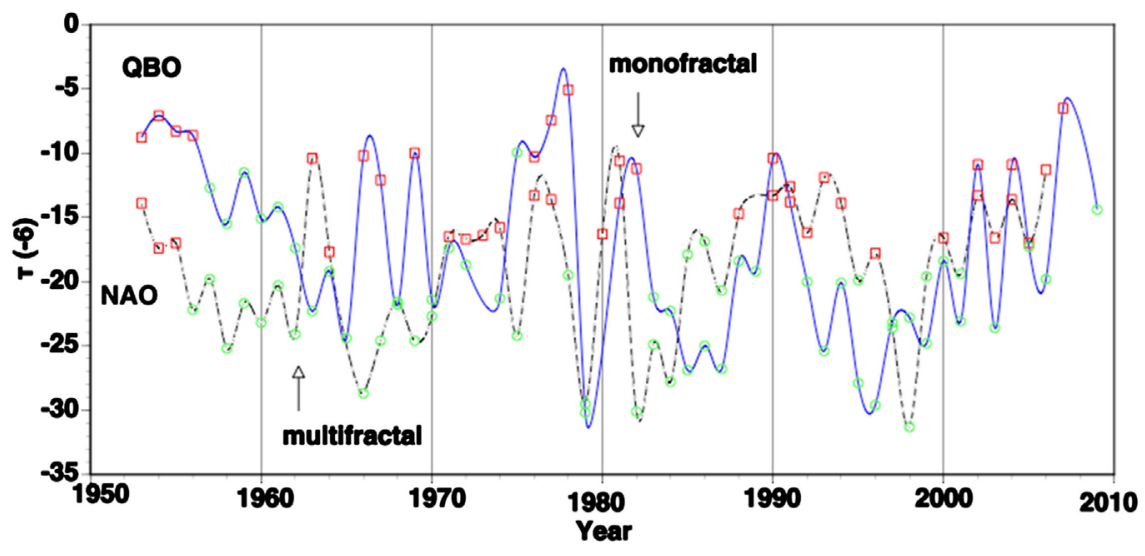

Wavelet Coherence
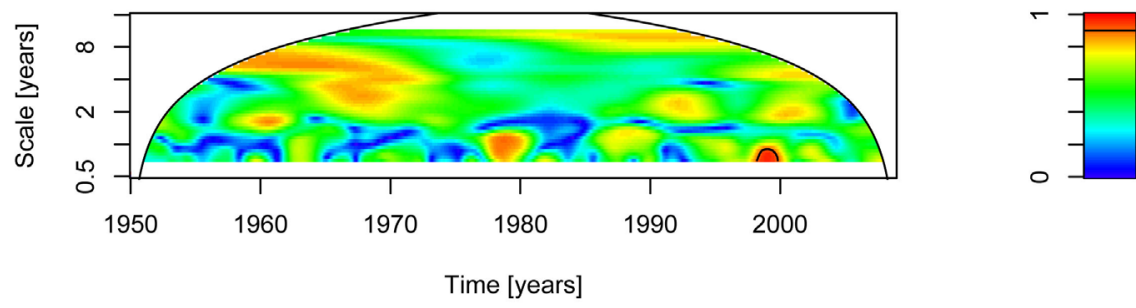

Phase
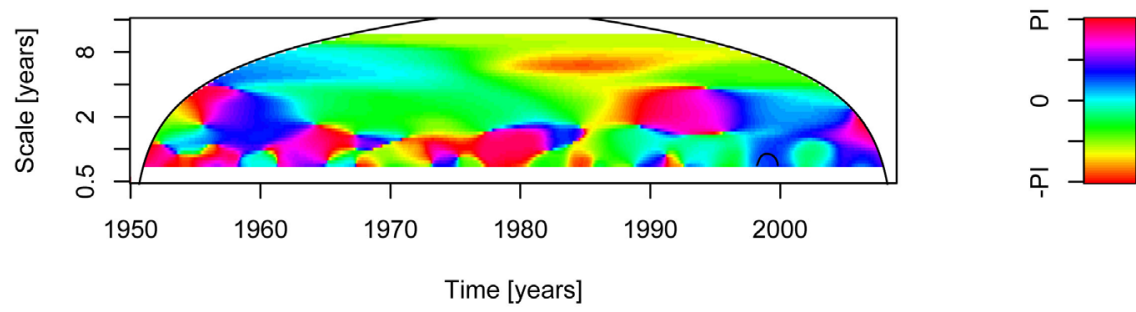

Figure 5. The $t(-6)$ of the NAO and QBO indices (top). Wavelet coherence (middle) and phase (bottom) between the NAO index and total QBO. In the wavelet phase, the positive value shown by the blue and pink shading means that the NAO leads the QBO and the negative value shown by the green, yellow and red shading means that QBO leads the NAO.

During the period 1985 and 2010, for the QBO and total ozone, the change of multifractality are very similar. The coherence between the QBO index and total ozone was strong in 2 - 3 year scale for 1980-1995 and in 1 year scale for 1995-2008 and the leads of the QBO index were observed. The influence of QBO on ozone has been shown and this result consisted with the result of Baldwin as below. Chemical constituents, such as ozone, water vapor, and methane, are affected by circulation changes induced by the QBO [11].

During the period 1950 and 2010, for the NAO and QBO, the change of multifractality is very similar. The coherence between the NAO and QBO indices was strong and the lead and delay of the QBO index was observed. Holton and Tan [14] have presented strong evidence that the equatorial QBO modulates the 

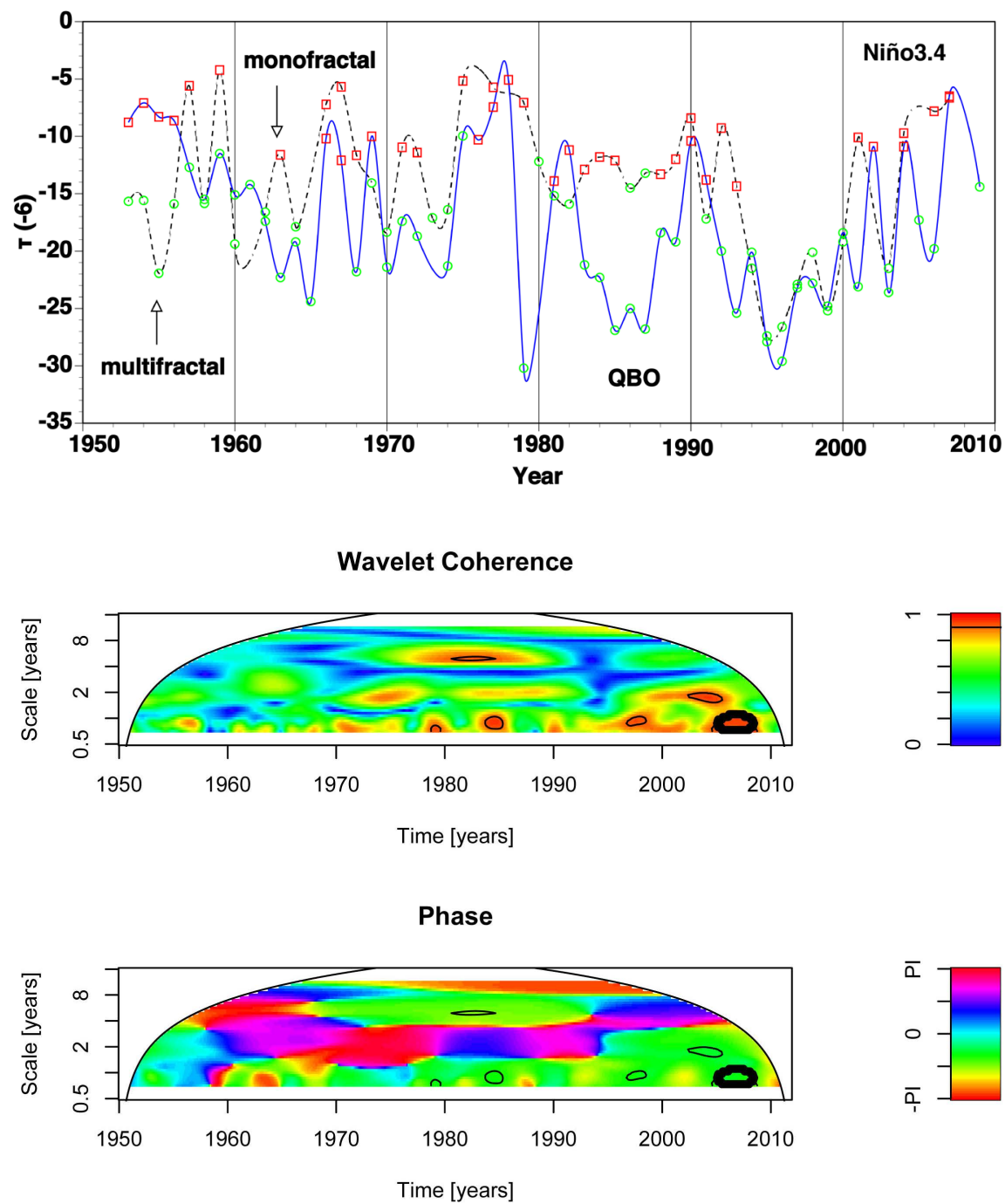

Figure 6. The $\tau(-6)$ of the Niño3.4 and QBO indices (top). Wavelet coherence (middle) and phase (bottom) between the Niño3.4 index and QBO. In the wavelet phase, the positive value shown by the blue and pink shading means that the Niño3.4 leads the QBO and the negative value shown by the green, yellow and red shading means that QBO leads the Niño3.4.

global circulation at $50 \mathrm{mb}$. The solar cycle modulation of the NAO is more strongly enhanced in the weaterly phase of the 50-hPa QBO wind [15]. On the other hand, a clear $\mathrm{AO} / \mathrm{NAO}$ response to the $\mathrm{QBO}$ is seen at the surface [14], with the tropospheric anomalies lagging the stratospheric anomalies by about 2 3 weeks [16].

During the period 1950 and 2010, for the Niño3.4 and QBO, the change of multifractality is very similar. The coherence between the Niño3.4 and QBO indices was strong and the lead and delay of the QBO index was observed. Between 1950 and 2010 years, for the SOI and QBO, the change of multifractality is very similar. The coherence between the SOI and QBO index was strong and the lead and delay of the QBO index was observed. During the period 1950 and 2010, especially for the 1970s, for the Niño3.4 and SOI, the matching in monofractality 

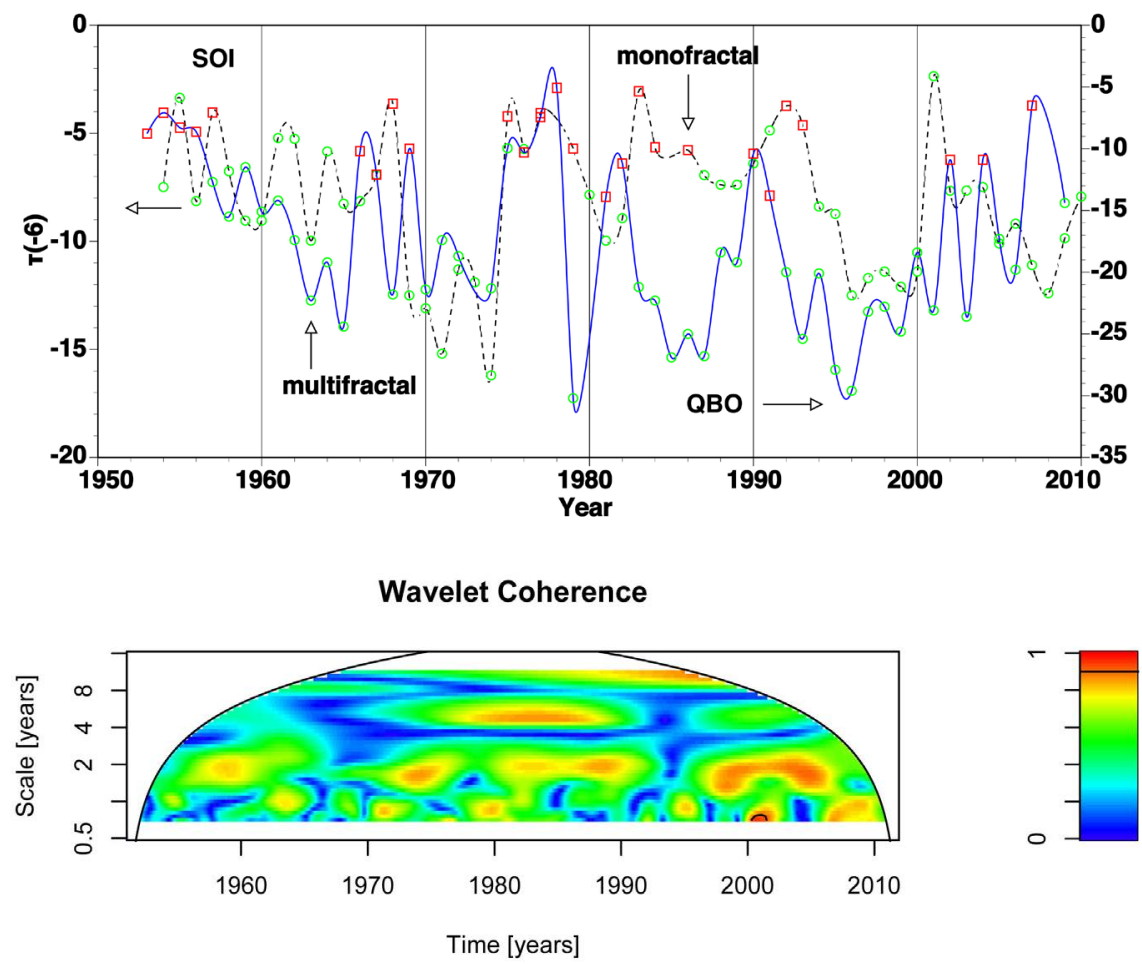

Phase
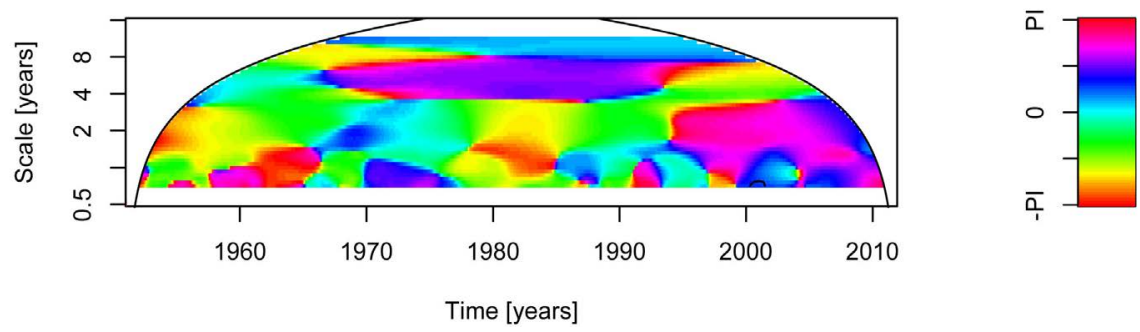

Figure 7. The $t(-6)$ of the SOI and QBO index (top). Wavelet coherence (middle) and phase (bottom) between the SOI and QBO. In the wavelet phase, the positive value shown by the blue and pink shading means that the SOI leads the QBO and the negative value shown by the green, yellow and red shading means that QBO leads the SOI.

or multifractality is observed and the increase and decrease of multifractality are very similar, that is the change of multifractality are very similar. The multifractal analysis used in this study is effective as a method to analyze complicated time series data. The relation between the QBO and ENSO has been shown. During the east phase of the QBO (QBOE), the Pacific regional pressure and circulation anomalies which arise in response to QBO-linked trends in convective activity are consistent with conditions leading to warm ENSO events (El Niño). If the heat content of the warm pool is sufficient, a warm event will occur. Conversely, conditions favoring the development of cold events (La Nina) tend to occur in association with the westerly phase of the QBO (QBOW) [17]. On the other hand, ENSO modulates QBO properties and the underlying easterly jet in QBOW is weaker during El Niño compared to La Nina, while the underlying 
westerly jet in QBOE is stronger during El Niño compared to La Nina [18].

We show the absolute value of $t(-6)$ for QBO, NAO, and Niño3.4 in Figure 8. The vertical axis is natural logarithmically transformed. The change rate for $\mathrm{NAO}$ and QBO was similar. Especially, the change rate for Niño3.4 index was large between 1950 and 1980 and after the 1976/77 regime shift, the change rate for Niño3.4 became small and ENSO became stable. The negative large values of the $\tau(-6)$ show large multifractality and instability. The absolute value of $t(-6)$ for NAO was large, hence the multifractality for NAO was strong. At some period, the absolute value of $\tau(-6)$ for $\mathrm{QBO}$ was small, hence the multifractality for QBO was weak. During the period 1950 and 1980, the absolute value of $\tau(-6)$ for Niño3.4 was small, hence the multifractality for Niño3.4 was weak. Hence the multifractality for NAO and QBO was strong comparing to Niño3.4 and the change of NAO and QBO was unstable.

The relation among the solar activity, total ozone, QBO, NAO, and ENSO were shown clearly by the wavelet coherence and phase and the similarity of the change of multifractality.

\section{Conclusions}

We investigated the change of multifractal behavior of the climate index. We used the wavelet transform to analyze the multifractal behavior of the F10.7 flux, total ozone, QBO, NAO, and Niño3.4 indices. We showed the change of multifractality by plotting the $\tau(-6)$. In this study, we examined the influence of the solar activity on the climate through the total ozone, and QBO. To detect the changes of multifractality, we examined the multifractal analysis using the wavelet transform on the F10.7 flux, total ozone, NAO, and QBO indices. Moreover we investigated the wavelet coherence and phase of these indices.

During the period 1950 and 2010, for the F10.7 flux and QBO index, the matching in monofractality or multifractality is observed and the increase and decrease of multifractality are similar; that is the change of multifractality is similar. In the same way, it is very similar, during the period 1985 and 2010, for

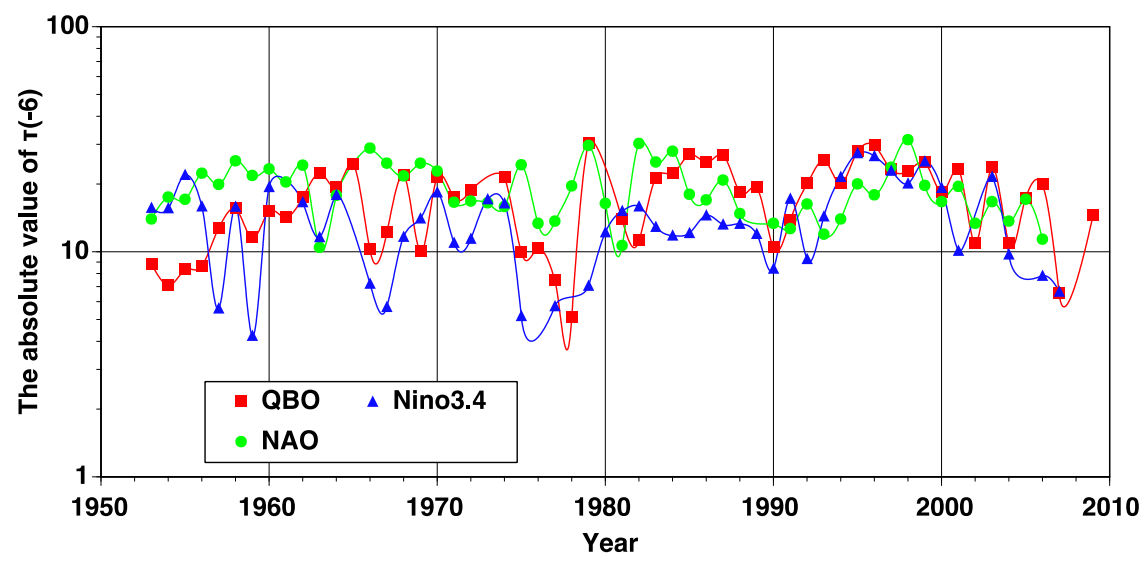

Figure 8. The absolute value of $t(-6)$ for QBO, Niño3.4, and NAO indices. The vertical axis is natural logarithmically transformed. 
the QBO and the total ozone, and during the period 1950 and 2010, for the QBO and, NAO and for the QBO, and Niño3.4. Compared to Niño3.4, the multifractality of NAO and $\mathrm{QBO}$ was strong and it turns out that they are undergoing unstable change.

The relation among the solar activity, total ozone, QBO, NAO, and ENSO was clarified by the methods of fractal analysis and the wavelet coherence. These findings will contribute to the research of the relation between the solar activity and climate change.

\section{References}

[1] Svensson, C., Olsson, J. and Berndtsson, R. (1996) Multifractal Properties of Daily Rainfall in Two Different Climates. Water Resources Research, 32, 2463-2472. https://doi.org/10.1029/96WR01099

[2] Muzy, J.F., Bacry, E. and Arneodo, A. (1991) Wavelets and Multifractal Formalism for Singular Signals: Application to Turbulence Data. Physical Review Letters, 67, 3515-3518. https://doi.org/10.1103/PhysRevLett.67.3515

[3] Maruyama, F., Kai, K. and Morimoto, H. (2015) Wavelet-Based Multifractal Analysis on Climatic Regime Shifts. Journal of the Meteorological Society of Japan, 93, 331-341. https://doi.org/10.2151/jmsj.2015-018

[4] Lean, J.L., Rottman, G.L., Kyle, H.L., Woods, T.N., Hickey, J.R. and Puga, L.C. (1997) Detection and Parameterization of Variations in Solar Mid and Near Ultraviolet Radiation (200 to $400 \mathrm{~nm}$ ). Journal of Geophysical Research, 102, 29939-29956. https://doi.org/10.1029/97JD02092

[5] Kodera, K. and Kuroda, Y. (2002) Dynamical Response to the Solar Cycle. Journal of Geophysical Research, 107, 4749. https://doi.org/10.1029/2002JD002224

[6] Hood, L.L., Soukharev, B.E. and McCormack, J.P. (2010) Decadal Variability of the Tropical Stratosphere: Secondary Influence of the El Niño-Southern Oscillation. Journal of Geophysical Research, 115, D11113. https://doi.org/10.1029/2009JD012291

[7] Randell, W.J., Garcia, R.R., Calvo, N. and Marsh, D. (2009) ENSO Influence on Zonal Mean Temperature and Ozone in the Tropical Lower Stratosphere. Geophysical Research Letters, 36, L15822. https://doi.org/10.1029/2009GL039343

[8] Gray, L.J., Crooks, S., Pascoe, C., Sparrow, S. and Palmer, M. (2004) Solar and QBO Influences on the Timing of Stratospheric Sudden Warmings. Journal of the Atmospheric Sciences, 61, 2777-2796. https://doi.org/10.1175/JAS-3297.1

[9] Alexander, R. and Feynman, J. (2002) Solar Influence on a Major Mode of Atmospheric Variability. Journal of Geophysical Research, 107, 4209. https://doi.org/10.1029/2001JD001239

[10] McCormack, J.P. (2003) The Influence of the 11-year Solar Cycle on the Quasi-Biennial Oscillation. Geophysical Research Letters, 30, 2162. https://doi.org/10.1029/2003GL018314

[11] Baldwin, M.P., Gray, L.J., Dunkerton, T., Hamilton, J., Haynes, K., Randel, P.H., Holton, W.J., Alexander, J.R., Hirota, M.J., Horinouchi, I., Jones, T., Kinnersley, D.B.A., Marquardt, J.S., Sato, C. and Takahashi, M. (2001) The Quasi-Biennial Oscillation. Reviews of Geophysics, 39, 179-229. https://doi.org/10.1029/1999RG000073

[12] Boberg, F. and Lundstedt, H. (2003) Solar Wind Electric Field Modulation of the 
NAO: A Correlation Analysis in the Lower Atmosphere. Geophysical Research Letters, 30. https://doi.org/10.1029/2003GL017360

[13] Frish, U. and Parisi, G. (1985) On the Singularity Structure of Fully Developed Turbulence. In: Ghil, M., Benzi, R. and Parisi, G., Eds., Turbulence and Predictability in Geophysical Fluid Dynamics and Climate Dynamics, North-Holland, New York, 84-88.

[14] Holton, J.R. and Tan, H.C. (1980) The Influence of the Equatorial Quasi-Biennial Oscillation on the Global Circulation at $50 \mathrm{mb}$. Journal of the Atmospheric Sciences, 37, 2200-2208. https://doi.org/10.1175/1520-0469(1980)037<2200:TIOTEQ >2.0.CO;2

[15] Kuroda, Y. (2007) Effect of QBO and ENSO on the Solar Cycle Modulation of Winter North Atlantic Oscillation. Journal of the Meteorological Society of Japan, 85, 889-898. https://doi.org/10.2151/jmsj.85.889

[16] Baldwin, M.P. and Dunkerton, T.J. (1999) Propagation of the Arctic Oscillation from the Stratosphere to the Troposphere. Journal of Geophysical Research, 104, 30937-30946. https://doi.org/10.1029/1999JD900445

[17] Gray, W.M., Sheaffer, J.D. and Knaff, J.A. (1992) Influence of the Stratospheric QBO on ENSO Variability. Journal of the Meteorological Society of Japan, 70, 975-994. https://doi.org/10.2151/jmsj1965.70.5_975

[18] Schirber, S. (2014) Influence of ENSO on the QBO: Results from an Ensemble of Idealized Simulations. Journal of Geophysical Research: Atmospheres. 